\title{
Cone beam computed tomography (CBCT) in periodontal diseases: a Systematic review based on the efficacy model
}

\author{
Hassan Assiri ${ }^{1 *}$, Ali Azhar Dawasaz ${ }^{1}$, Ahmad Alahmari $^{2}$ and Zuhair Asiri ${ }^{3}$
}

\begin{abstract}
Background: Periodontal diseases are prevalent among adult populations. Its diagnosis depends mainly on clinical findings supported by radiographic examinations. In previous decades, cone beam computed tomography has been introduced to the dental field. The aim of this study was to address the diagnostic efficacy of cone-beam computed tomographic (CBCT) imaging in periodontics based on a systematic search and analysis of the literature using the hierarchical efficacy model.
\end{abstract}

Methods: A systematic search of electronic databases such as PubMed, Scopus, Web of Science, and Cochrane was conducted in February 2019 to identify studies addressing the efficacy of CBCT imaging in Periodontics. The identified studies were subjected to pre-identified inclusion criteria followed by an analysis using a hierarchical model of efficacy (model) designed for an appraisal of the literature on diagnostic imaging modality. Four examiners performed the eligibility and quality assessment of relevant studies and consensus was reached in cases where disagreement occurred.

Results: The search resulted in 64 studies. Of these, 34 publications were allocated to the relevant level of efficacy and quality assessments wherever applicable. The overall diagnostic accuracy of the included studies showed a low or moderate risk of bias and applicability concerns in the use of CBCT. In addition, CBCT is accurate in identifying periodontal defects when compared to other modalities. The studies on the level of patient outcomes agreed that CBCT is a reliable tool for the assessment of outcomes after the treatment of periodontal defects.

Conclusion: $\mathrm{CBCT}$ was found to be beneficial and accurate in cases of infra-bony defects and furcation involvements.

Keywords: Cone-beam computed tomography, Digital volume tomography, Furcation defects, Infra-bony defects, Periodontitis

\section{Background}

Periodontal diseases affect the structures surrounding the teeth [1-3]. They range from the mildest form of gingivitis to the most aggressive form of periodontitis. Gingivitis is limited to the inflammation of gingiva without deep

\footnotetext{
* Correspondence: halmuawad@kku.edu.sa

${ }^{1}$ Department of Oral Biology and Diagnostic Science, King Khalid University, College of Dentistry, Abha, Saudi Arabia

Full list of author information is available at the end of the article
}

involvement of teeth-supporting structures such as the alveolar bone. On the other hand, periodontitis does extend to the alveolar bone [4-7]. It starts with the formation of a periodontal pocket and, consequently, if not treated, leads to bone and tooth loss. Another manifestation of the periodontal diseases in molar-premolar teeth is the formation of furcation defects [8-11]. Since gingivitis affects only the soft tissue, its diagnosis and treatment rely solely on clinical findings including redness, puffiness, and bleeding

(c) The Author(s). 2020 Open Access This article is licensed under a Creative Commons Attribution 4.0 International License which permits use, sharing, adaptation, distribution and reproduction in any medium or format, as long as you give appropriate credit to the original author(s) and the source, provide a link to the Creative Commons licence, and indicate if changes were made. The images or other third party material in this article are included in the article's Creative Commons licence, unless indicated otherwise in a credit line to the material. If material is not included in the article's Creative Commons licence and your intended use is not permitted by statutory regulation or exceeds the permitted use, you will need to obtain permission directly from the copyright holder. To view a copy of this licence, visit http://creativecommons.org/licenses/by/4.0/ The Creative Commons Public Domain Dedication waiver (http://creativecommons.org/publicdomain/zero/1.0/) applies to the data made available in this article, unless otherwise stated in a credit line to the data. 
[12-14]. However, periodontitis could lead to bone resorption depending on its severity; hence, its diagnosis and treatment planning relies on clinical methods supported by radiographic imaging [15-17].

There are several risks to using clinical examination alone, which could prevent the accurate diagnosis of periodontitis, including gingival tissue consistency, inflammation severity, pressure while probing, probe size, probing angulation, and dental restoration existence [18, 19]. In dental practice, practitioners routinely utilize conventional radiography such as periapical, bitewing, and panoramic $x$-ray to evaluate the bone loss and overall condition of the periodontal disease [18]. Nevertheless, the twodimensional $\mathrm{x}$-ray has some limitations, mainly due to the overlapping of structures [20]. Thus, the detection of bone craters, inter-radicular bone loss, and lingual and buccal marginal bone loss necessitate the consideration of threedimensional radiography [17, 21-24].

Cone-beam computed tomography (CBCT) has been used frequently in the last two decades in dentomaxillofacial region [25]. It has many advantages compared to conventional computed tomography (CT) including low price, low radiation dose, and ease of accommodation at dental offices [25-27]. In addition, it has the ability to view the structures in three dimensions [28-30]. CBCT images of periodontal bone lesions offer a highly informative value. The spatial representation of the alveolar bone in all three planes has a significant role in periodontology, as treatment decisions and long-term prognosis rely on it [11]. Accordingly, it can play a potential role as an adjunct to clinical examination in the case of periodontal diseases [28, 31, 32].

Evidence-based dentistry aims to identify the best available evidence to justify the efficacy and use of any dental imaging or test in actual practice. Accordingly, Fryback and Thornbury came up with a hierarchal model of efficacy in the early nineties to sort out the best available evidence for a diagnostic tool [33].

There are several published studies on the role of CBCT in periodontal diseases in the literature [13-15].

However, the extent to which CBCT is efficient and accurate in the diagnosis, treatment planning, decisionmaking, and treatment outcomes of periodontal diseases remains ambiguous. On the path to routine use, especially under consideration of higher radiation exposure to patients, the gain in additional information of clinical relevance has to be explored and evaluated. Consequently, we conducted a systematic review to address the efficacy of $\mathrm{CBCT}$ in periodontal diseases.

\section{Methods}

This review was conducted based on guidelines from Preferred Reporting Items for Systematic Review and Meta-Analysis (PRISMA) [34] and guidance from the center for reviews and dissemination (CRD) for undertaking a systematic review in health care [35]. The eligibility criteria for inclusion and exclusion were set. Then, the included studies were assigned to the suitable level of efficacy. In the meantime, the review question was designed according to the PICO (Population, or Problem, Intervention or Exposure, Comparison, Outcome) element [36]. Finally, each study was evaluated for quality using the predetermined tool for quality assessment (QUADAS 2).

\section{Criteria for inclusion}

I. Original studies

II. Systematic reviews

III. The study must assess the role of CBCT in plaqueinduced periodontal disease

IV. Each study can be on any level of the efficacy model [33]

V. Studies addressing CBCT accuracy should compare it to clinical or radiographic measurements

\section{Criteria for exclusion}

I. Case reports

II. Narrative reviews

III. Languages other than English

IV. Studies addressing periapical periodontitis caused by pulpal infection

V. Studies addressing the bone status for the purpose of dental implant

VI. Studies highlighting the use of CBCT to address artificially created bone defects

\section{- Problem specification:}

The research question was defined as "what is the diagnostic efficacy of CBCT in individuals with periodontal diseases?"

\section{- Literature search:}

Four databases PubMed, Scopus, Cochrane, and Web of Science were searched till February 2019 to identify the relevant studies. The search strategy is shown in Table 1.

\section{- Study retrieval:}

The resultant studies were subjected to a duplicate check on the RefWorks database. The studies were then reviewed by four authors for relevance based on 
Table 1 Study search strategy

\begin{tabular}{|c|c|c|}
\hline Database and its supplies & Index terms & Results \\
\hline $\begin{array}{l}\text { Pubmed (US National Library of } \\
\text { Medicine (NLM)) }\end{array}$ & $\begin{array}{l}\text { Cone beam computed tomography [MeSH]) OR Cone beam computed tomography } \\
\text { [Title/Abstract]) OR Cone beam CT [Title/Abstract]) OR CBCT [Title/Abstract]) OR } \\
\text { Dental computed tomography [Title/Abstract]) OR Digital volume tomography } \\
\text { [Title/Abstract]) OR Volumetric tomography [Title/Abstract]) OR 3D image } \\
\text { [Title/Abstract]) OR Three dimensional imaging [Title/Abstract]) OR Imaging, three } \\
\text { dimensional [MeSH] AND Periodontitis [MeSH] OR Periodontal disease [Title/Abstract]) } \\
\text { OR Periodontal diseases [Title/Abstract]) OR Furcation defects [MeSH]) OR Furcation } \\
\text { involvement [Title/Abstract]) OR Alveolar bone loss [MeSH]) OR Intrabony defect } \\
\text { [Title/Abstract] }\end{array}$ & 539 \\
\hline Scopus (Elsevier) & $\begin{array}{l}\text { TITLE-ABS-KEY (Cone beam computed tomography) or TITLE-ABS-KEY (Cone beam } \\
\text { computed tomography) or TITLE-ABS-KEY (Cone beam CT) or TITLE-ABS-KEY (CBCT) } \\
\text { or TITLE-ABS-KEY (Dental computed tomography) or TITLE-ABS-KEY (Digital volume } \\
\text { tomography) or TITLE-ABS-KEY (Volumetric tomography) or TITLE-ABS-KEY(3D image) } \\
\text { or TITLE-ABS-KEY (Three-dimensional imaging) AND TITLE-ABS-KEY (Periodontitis) or } \\
\text { TITLE-ABS-KEY (Periodontal disease) or TITLE-ABS-KEY (Periodontal diseases) or } \\
\text { TITLE-ABS-KEY (Furcation involvement) or TITLE-ABS-KEY (Furcation defects) or } \\
\text { TITLE-ABS-KEY (Intrabony defect) }\end{array}$ & 746 \\
\hline Cochrane (Wiley InterScience) & $\begin{array}{l}\text { Cone beam computed tomography [MeSH] or Cone beam computed tomography } \\
\text { (word variations ti, ab, kw) or Cone beam CT (word variations ti, ab, kw) or CBCT } \\
\text { (word variations ti, ab, kw) or Dental computed tomography (word variations ti, ab, } \\
\text { kw) or Digital volume tomography (word variations ti, ab, kw) or Volumetric } \\
\text { tomography (word variations ti, ab, kw) or 3D image (word variations ti, ab, kw) or } \\
\text { lamging, three dimensional [MeSH] or AND Periodontitis [MeSH] or Periodontal } \\
\text { disease (word variations ti, ab, kw) or Periodontal diseases (word variations ti, ab, kw) } \\
\text { or Furcation involvement (word variations ti, ab, kw) or Furcation defects [MeSH] or } \\
\text { Alveolar bone loss [MeSH] or Intrabony defect (word variations ti, ab, kw) }\end{array}$ & 71 \\
\hline Web of Science & $\begin{array}{l}\text { Cone beam computed tomography (Topic) or Cone beam CT (Topic) or CBCT (Topic) } \\
\text { or Dental computed tomography (Topic) or Digital volume tomography (Topic) or } \\
\text { Volumetric tomography (Topic) or 3D image (Topic) or Three dimensional imaging } \\
\text { (Topic) or AND Periodontitis (Topic) or Periodontal disease (Topic) or Periodontal } \\
\text { diseases (Topic) or Furcation defects (Topic) or Furcation involvement (Topic) or } \\
\text { Alveolar bone loss (Topic) or Intrabony defect (Topic) }\end{array}$ & 555 \\
\hline
\end{tabular}

inclusion and exclusion criteria. After that, the studies meeting the eligibility criteria were assigned for full-text screening. Where uncertainty was present, discussions were conducted between the authors to reach an agreement on whether to include or exclude a study based on the predefined inclusion and exclusion criteria.

- Data extraction \& quality assessment: Finally, each of the selected studies was assigned for data extraction and analysis. After that, each study was allocated its suitable level of efficacy. A Revised Tool for the Quality Assessment of Diagnostic Accuracy Studies (QUADAS 2) was used for quality assessment. This tool contains four domains: patient selection, index test, reference standard, and flow and timing. Each domain is assessed in terms of risk of bias and the first three domains are assessed in terms of concerns regarding applicability.

Signaling questions are included to help judge the risk of bias [37].

\section{Result}

\section{Studies allocation}

The search strategy of the four databases yielded 1717 articles: PubMed 539, Scopus 746, Cochrane 71, and
Web of Science 555. After a duplicate check using RefWorks, the result came up to 1262 . These were subjected to the title and abstract screening by the two authors. A set of 65 studies were linked to the full-text review. A total of 28 articles were excluded because they did not possess at least one of the inclusion criteria. Studies reported by $[28,38-50]$ were ex vivo studies and out of our review.

Plaque-induced periodontitis was not addressed, therefore, studies on that issue were excluded. In addition, studies that did not belong to any level of efficacy were disregarded [51-55]. Studies that addressed bone density conducted by Al Zahrani et al. [56] and bone coverage conducted by Ferriera et al. [57] were also excluded. Published studies by Evangelista et al. [58], Sun et al. [59], and Leung et al. [60] discussed only the naturally occurring dehiscence and fenestration, hence, they were disregarded. Studies reported by Goodarzy et al. [61] and Nagao et al. [62] were excluded because they did not include patients having periodontitis. The case report presented by Naitoh et al. [63] was disregarded as well. Studies published in languages other than English; reported by Deng et al. [64]) was excluded. Figure 1 shows the results for systematic reviews according to the PRISMA flow chart. Table 2 shows the studies that were included and their suitable efficacy level. 

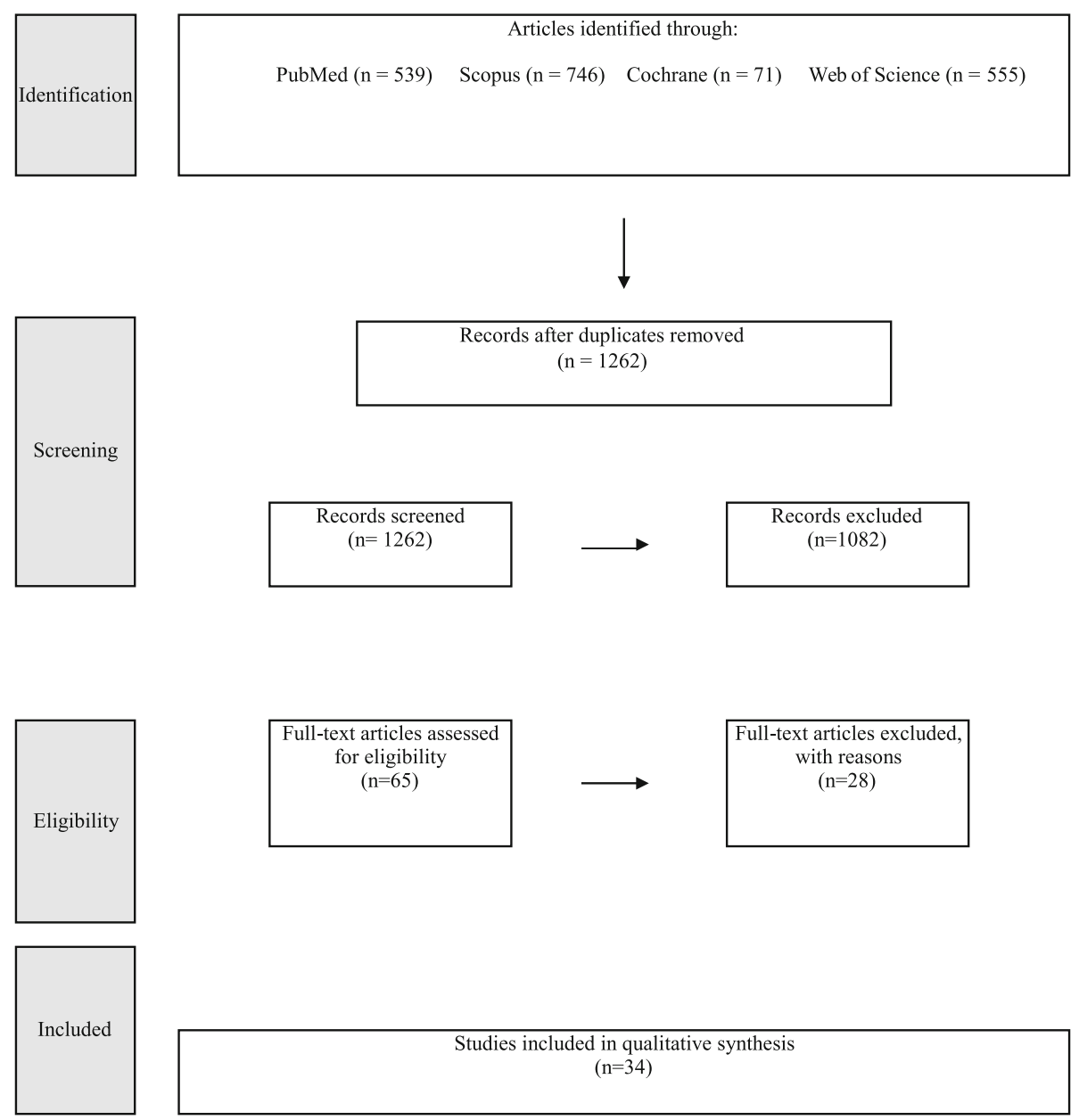

Fig. 1 The results for systematic reviews according to the PRISMA flow chart

- Quality assessment

After allocating each study its suitable efficacy level, special tools of quality assessment were used for each one as described in the literature [37].

- Technical efficacy studies:

There was no study identified on this level of efficacy.

- Diagnostic accuracy studies:

The results revealed eighteen studies $[65,69,71,74$, $76,81,84,86-96]$ on diagnostic accuracy. The QUADAS-2 assessment tool was used for quality assessment [34]. Table 3 reveals the results of the quality assessment using QUADAS-2.

There were three studies that included a previously published systematic, manual search of the reference lists of the included articles [64, 81, 87], among which one study by Deng et al. [64] was found to be published in a Chinese language and hence excluded.

\section{- Diagnostic thinking efficacy:}

Only one study was found to be on the level of diagnostic thinking efficacy [66]. The author investigated the effect of $\mathrm{CBCT}$ on the treatment decision-making after taking into consideration the clinical parameters.

- Therapeutic efficacy:

In this level of efficacy, only one study, Pajnigara et al. [67], seemed relevant.

- Patient outcome efficacy:

Our research resulted in eight studies in which CBCT was used to address the patients' outcomes in relation to periodontal disease. All of the studies are randomized clinical trials [68, 72, 75, 77, 79, 82, 85]. Table 4, the CASP (critical appraisal skills program) checklist, was used to assess outcomes.

- Societal efficacy:

Only one study was found to be relevant in this level of efficacy, Walter et al. [69]. The quality assessment was done using the QUADAS 2 tool.

\section{Systematic reviews}

The remaining six studies $[6,70,73,78,80,83]$ were found to be systematic reviews for which the AMSTAR- 
Table 2 Studies that were included and their suitable efficacy level

\begin{tabular}{|c|c|c|c|c|c|c|c|}
\hline \multicolumn{6}{|l|}{ Included studies } & \multirow{3}{*}{$\begin{array}{l}\text { Excluded } \\
\text { studies }\end{array}$} & \multirow[t]{3}{*}{ Exclusion reason } \\
\hline \multicolumn{5}{|l|}{ Efficacy levels } & \multirow{2}{*}{$\begin{array}{l}\text { Systematic } \\
\text { Review }\end{array}$} & & \\
\hline 12 & 3 & 4 & 5 & 6 & & & \\
\hline $\begin{array}{l}\text { Nagao et al. } \\
2006 \text { [65] }\end{array}$ & $\begin{array}{l}\text { Walter et } \\
\text { al. } 2009 \\
\text { [66] }\end{array}$ & $\begin{array}{l}\text { Pajnigara et } \\
\text { al. } 2016 \\
{[67]}\end{array}$ & $\begin{array}{l}\text { Grimrad et } \\
\text { al.2009 [68] }\end{array}$ & $\begin{array}{l}\text { Walter et } \\
\text { al. } 2012 \\
\text { [69] }\end{array}$ & $\begin{array}{l}\text { Walter et } \\
\text { al.2016 [70] }\end{array}$ & $\begin{array}{l}\text { Micsh et al. } \\
2006[42]\end{array}$ & Ex vivo \\
\hline \multicolumn{2}{|l|}{$\begin{array}{l}\text { Walter et al. } \\
2010[71]\end{array}$} & & $\begin{array}{l}\text { Gupta SJ et } \\
\text { al. } 2014 \text { [72] }\end{array}$ & & $\begin{array}{l}\text { Anter et } \\
\text { al.2016 [73] }\end{array}$ & $\begin{array}{l}\text { Naitoh et al. } \\
2006 \text { [63] }\end{array}$ & Case report \\
\hline \multicolumn{2}{|l|}{$\begin{array}{l}\text { De Faria et } \\
\text { al.2012 [74] }\end{array}$} & & $\begin{array}{l}\text { Khosropana } \\
\text { et al.2015 } \\
{[75]}\end{array}$ & & $\begin{array}{l}\text { Nikolic- } \\
\text { Jakoba et } \\
\text { al.2016 [6] }\end{array}$ & $\begin{array}{l}\text { Nagao et } \\
\text { al.2007 [62] }\end{array}$ & Did no check for periodontitis \\
\hline \multicolumn{2}{|l|}{$\begin{array}{l}\text { Fiejo et al. } 2012 \\
\text { [76] }\end{array}$} & & $\begin{array}{l}\text { Bhavsar et } \\
\text { al. } 2016 \text { [77] }\end{array}$ & & $\begin{array}{l}\text { Haas et al. } \\
2018 \text { [78] }\end{array}$ & $\begin{array}{l}\text { Vandenberghe } \\
\text { et al. } 2007 \text { [47] }\end{array}$ & Ex vivo \\
\hline \multicolumn{2}{|l|}{$\begin{array}{l}\text { Walter et al. } \\
2012[69]\end{array}$} & & $\begin{array}{l}\text { Pajnigara et } \\
\text { al. } 2017 \text { [79] }\end{array}$ & & $\begin{array}{l}\text { Choi et al. } \\
2018 \text { [80] }\end{array}$ & $\begin{array}{l}\text { Mol et al. } 2008 \\
\text { [43] }\end{array}$ & Ex vivo \\
\hline \multicolumn{2}{|l|}{$\begin{array}{l}\text { Raichur et al. } \\
2012 \text { [81] }\end{array}$} & & $\begin{array}{l}\text { Dutra et al. } \\
2017 \text { [82] }\end{array}$ & & $\begin{array}{l}\text { Woelber et } \\
\text { al. } 2018 \text { [83] }\end{array}$ & $\begin{array}{l}\text { Vandenberghe } \\
\text { et al. } 2008 \text { [46] }\end{array}$ & Ex vivo \\
\hline \multicolumn{2}{|l|}{$\begin{array}{l}\text { Marinescu et al. } \\
2013 \text { [84] }\end{array}$} & & $\begin{array}{l}\text { Nemoto et } \\
\text { al. } 2018 \text { [85] }\end{array}$ & & & $\begin{array}{l}\text { Noujeim et al. } \\
2009 \text { [44] }\end{array}$ & Ex vivo \\
\hline \multicolumn{2}{|l|}{$\begin{array}{l}\text { Qiao et al. } 2013 \\
\text { [86] }\end{array}$} & & & & & $\begin{array}{l}\text { Leung et al. } \\
2010[60]\end{array}$ & Ex vivo \\
\hline \multicolumn{2}{|l|}{$\begin{array}{l}\text { Haghgoo et } \\
\text { al.2014 [87] }\end{array}$} & & & & & $\begin{array}{l}\text { Evangelista et } \\
\text { al.2010 [58] }\end{array}$ & $\begin{array}{l}\text { Addressed naturally occurring dehiscence } \\
\text { and fenestration in patients with } \\
\text { malocclusions }\end{array}$ \\
\hline \multicolumn{2}{|l|}{$\begin{array}{l}\text { Banodkar et al. } \\
2015 \text { [88] }\end{array}$} & & & & & $\begin{array}{l}\text { Ferreira et } \\
\text { al.2013 [57] }\end{array}$ & Study targets bone coverage \\
\hline \multicolumn{2}{|l|}{$\begin{array}{l}\text { Cimbaljevic et al. } \\
2015 \text { [89] }\end{array}$} & & & & & $\begin{array}{l}\text { DG Pour et al. } \\
2015 \text { [61] }\end{array}$ & $\begin{array}{l}\text { Does not involve patients with } \\
\text { periodontitis }\end{array}$ \\
\hline \multicolumn{2}{|l|}{$\begin{array}{l}\text { Darby et al. } \\
2015 \text { [90] }\end{array}$} & & & & & $\begin{array}{l}\text { AlShaer et al. } \\
2013 \text { [55] }\end{array}$ & Does not belong to any level of efficacy \\
\hline \multicolumn{2}{|l|}{$\begin{array}{l}\text { Li F et al. } 2015 \\
\text { [91] }\end{array}$} & & & & & $\begin{array}{l}\text { Fliener et al. } \\
2013 \text { [40] }\end{array}$ & Ex vivo \\
\hline \multicolumn{2}{|l|}{$\begin{array}{l}\text { Guo et al. } 2016 \\
\text { [92] }\end{array}$} & & & & & $\begin{array}{l}\text { Kamuroglu et } \\
\text { al. } 2013 \text { [41] }\end{array}$ & Ex vivo \\
\hline \multicolumn{2}{|l|}{$\begin{array}{l}\text { Zhu et al. } 2016 \\
\text { [93] }\end{array}$} & & & & & $\begin{array}{l}\text { Amorfini et } \\
\text { al.2014 [54] }\end{array}$ & Does not belong to any level of efficacy \\
\hline \multicolumn{2}{|l|}{$\begin{array}{l}\text { Suphanantachat } \\
\text { et al. } 2017 \text { [94] }\end{array}$} & & & & & $\begin{array}{l}\text { Sun L et al. } \\
2015 \text { [59] }\end{array}$ & $\begin{array}{l}\text { Excluded patients with periodontal } \\
\text { diseases }\end{array}$ \\
\hline \multicolumn{2}{|l|}{$\begin{array}{l}\text { Padmanabhan } \\
\text { et al. } 2017 \text { [95] }\end{array}$} & & & & & $\begin{array}{l}\text { Yang et al. } \\
2015 \text { [53] }\end{array}$ & Does not belong to any level of efficacy \\
\hline \multirow[t]{8}{*}{$\begin{array}{l}\text { Zhang et al. } \\
2018 \text { [96] }\end{array}$} & & & & & & $\begin{array}{l}\text { Bagis et al. } \\
2015[38]\end{array}$ & Ex vivo \\
\hline & & & & & & $\begin{array}{l}\text { Takeshita et al. } \\
2015 \text { [28] }\end{array}$ & Ex vivo \\
\hline & & & & & & $\begin{array}{l}\text { Kolsuz et al. } \\
2015 \text { [48] }\end{array}$ & Ex vivo \\
\hline & & & & & & $\begin{array}{l}\text { Kamburoğlu et } \\
\text { al. } 2015 \text { [49] }\end{array}$ & $\begin{array}{l}\text { Animal study and not plaque-induced } \\
\text { periodontitis }\end{array}$ \\
\hline & & & & & & $\begin{array}{l}\text { Deng et al. } \\
2015 \text { [64] }\end{array}$ & Not in English Language \\
\hline & & & & & & $\begin{array}{l}\text { Lim et al. } 2016 \\
\text { [51] }\end{array}$ & Does not belong to any level of efficacy \\
\hline & & & & & & $\begin{array}{l}\text { Al-Zahrani et } \\
\text { al. } 2017 \text { [56] }\end{array}$ & $\begin{array}{l}\text { Targets bone density in aggressive } \\
\text { periodontitis and normal patients }\end{array}$ \\
\hline & & & & & & Ozcan et & Does not belong to any level of efficacy \\
\hline
\end{tabular}


Table 2 Studies that were included and their suitable efficacy level (Continued)

\begin{tabular}{|c|c|c|c|c|c|c|c|}
\hline \multicolumn{6}{|c|}{ Included studies } & \multirow{3}{*}{$\begin{array}{l}\text { Excluded } \\
\text { studies }\end{array}$} & \multirow[t]{3}{*}{ Exclusion reason } \\
\hline \multicolumn{5}{|c|}{ Efficacy levels } & \multirow{2}{*}{$\begin{array}{l}\text { Systematic } \\
\text { Review }\end{array}$} & & \\
\hline \multirow[t]{5}{*}{12} & 3 & 4 & 5 & 6 & & & \\
\hline & & & & & & al.2017 [52] & \\
\hline & & & & & & $\begin{array}{l}\text { Almeida et } \\
\text { al.2017 [39] }\end{array}$ & Ex vivo \\
\hline & & & & & & $\begin{array}{l}\text { Salineiro et } \\
\text { al.2017 [45] }\end{array}$ & Ex vivo \\
\hline & & & & & & $\begin{array}{l}\text { Elashiry et al. } \\
2018 \text { [50] }\end{array}$ & Not plaque-induced periodontitis \\
\hline
\end{tabular}

2 assessment tool [97] was used. It is a popular instrument modified from the original AMSTAR, which contains 16 checklist questions. (Refer to Table 5). The two authors meticulously screened each study in order to give a suitable answer for each checklist question.

\section{Discussion}

Alveolar bone loss is considered a primary symptom of periodontal diseases. Mostly, the assessment and treatment decisions depend on clinical measurements supported by conventional imaging modalities. However, 2D imaging has its own limitations for detecting bone defects, including overlapping. An estimation of bone loss buccolingually has led to the consideration of $3 \mathrm{D}$ imaging. However, to what extent the $\mathrm{CBCT}$ is effective in the diagnosis of periodontal diseases is not yet clear. Accordingly, our systematic review was designed to summarize the available evidence according to the hierarchal model of efficacy developed by Fryback et al. [33].

In our systematic review, we decided to exclude studies that are published in any language other than English because of time restriction. In addition, case reports and narrative reviews are considered in the literature as lowevidence studies. Studies addressing periapical conditions and implant-related periodontal problems were also excluded as they are beyond our aspect in this review. In the meantime, it was decided to not include studies conducted ex vivo where the periodontal defects are created artificially since we believe those results will not mimic the CBCT's performance when conducted on humans.

\section{Technical efficacy level}

It seems most of the studies conducted on the use of CBCT in periodontal disease were aimed at performance detection, accuracy estimation, or the treatment outcome assessment. The authors found no study reported in the literature dealt with the technical aspect of CBCT.

\section{Diagnostic accuracy level}

As mentioned earlier in this review, the QUADAS 2 tool was used for the quality assessment of diagnostic accuracy studies. Only studies conducted in vivo were included in this review. Some studies did not use explicit reference standards to compare CBCT with other modalities [71, 89, 90, 93, 94].

Cimbaljevic et al. [63] compared the periodontal probing with $\mathrm{CBCT}$ in the terms of furcation involvement in the absence of a reference standard. Likewise, Darby et al. [64] addressed the discrepancies in the clinical measurements obtained from patients' records with their available CBCT images. A study conducted by Suphaanantachat et al. [92] compared CBCT to conventional intraoral radiography. However, they did not use an actual reference standard for comparison. Similarly, Zhu J. et al. [86] has focused on the reproducibility of the different parameters of $\mathrm{CBCT}$ for the furcation involvement evaluation, and hence, no reference standard was used.

\section{Diagnostic thinking}

A study published by Walter et al. [66] on decisionmaking revealed discrepancies between clinically and CBCT-based therapeutic treatment approaches. The discrepancy was found after $59-82 \%$ of the teeth were investigated to find out whether less invasive or most invasive treatment should be considered. However, they concluded that CBCT provides informative details in cases of furcation involvement, and hence, it is considered a reliable tool in decision-making regarding treatment of furcation involvement.

\section{Therapeutic efficacy}

According to our interpretation and in correlation with the hierarchical model of efficacy [33], we found that the study conducted by Pajnigara et al. [67] fits on this level. They investigated the pre and post-surgical measurements of clinical and CBCT for furcation defects. Although they reported statistically significant differences between; clinical-presurgery CBCT $(P<0.0001,95 \% \mathrm{CI})$ 
Table 3 Quality assessment of diagnostic accuracy studies using QUADAS2 tool

\begin{tabular}{|c|c|c|c|c|c|c|c|c|c|c|}
\hline \multirow[t]{2}{*}{ Study } & \multirow[t]{2}{*}{ Sample } & \multirow[t]{2}{*}{ Index test } & \multirow{2}{*}{$\begin{array}{l}\text { Reference } \\
\text { standard } \\
\text { (comparator) }\end{array}$} & \multicolumn{4}{|c|}{ Risk of bias } & \multicolumn{3}{|c|}{ Applicability concern } \\
\hline & & & & $\begin{array}{l}\text { Patient } \\
\text { Selection }\end{array}$ & $\begin{array}{l}\text { Index } \\
\text { test }\end{array}$ & $\begin{array}{l}\text { Reference } \\
\text { test }\end{array}$ & $\begin{array}{l}\text { Flow and } \\
\text { timing }\end{array}$ & $\begin{array}{l}\text { Patient } \\
\text { selection }\end{array}$ & $\begin{array}{l}\text { Index } \\
\text { test }\end{array}$ & $\begin{array}{l}\text { Reference } \\
\text { standard }\end{array}$ \\
\hline $\begin{array}{l}\text { Nagao et al. } \\
2006[65]\end{array}$ & $\begin{array}{l}\text { Developing novel } \\
\text { method for } \\
\text { alveolar bone } \\
\text { resorption } \\
\text { assessment caused } \\
\text { by periodontitis }\end{array}$ & Dental CT & Not applicable & $\oplus$ & (:) & $\mathrm{N} / \mathrm{A}$ & (:) & 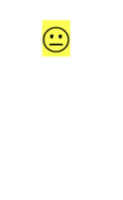 & 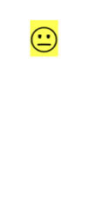 & $\mathrm{N} / \mathrm{A}$ \\
\hline $\begin{array}{l}\text { Walter et al. } \\
2010 \text { [71] }\end{array}$ & $\begin{array}{l}14 \text { patients, five } \\
\text { women and nine } \\
\text { men, with a mean } \\
\text { age of } 57.0 \text { years } \\
\text { (range } 42-81 \\
\text { years) and a } \\
\text { diagnosis of } \\
\text { generalized } \\
\text { chronic } \\
\text { periodontitis }\end{array}$ & $\begin{array}{l}\text { CBCT Accuitomo } \\
\text { with settings in the } \\
\text { range of } 74-90 \mathrm{kV} \text {, } \\
5-8 \mathrm{~mA} \text { and voxel } \\
\text { sizes in the range } \\
\text { of } 0.08-0.25 \mathrm{~mm}\end{array}$ & $\begin{array}{l}\text { Intrasurgical } \\
\text { measurements }\end{array}$ & (-) & (-) & (-) & (:) & (-) & (-) & (-) \\
\hline $\begin{array}{l}\text { De Faria et al. } \\
2012 \text { [74] }\end{array}$ & $\begin{array}{l}\text { Image records of } \\
39 \text { teeth from } 11 \\
\text { adult patients of } \\
\text { both genders, } \\
\text { aged between } 39 \\
\text { and } 66 \text { years }\end{array}$ & $\begin{array}{l}\text { CBCT i-CAT with } \\
120 \mathrm{kV} \text { and } 36.12 \\
\mathrm{mAs} \text {. The field of } \\
\text { view (FOV) was } 6 \\
\mathrm{~cm} \text { and the voxel } \\
\text { size was } 0.260 .260 .2 \\
\mathrm{~mm} \text { | Intraoral } \\
\text { radiography } \\
\text { (Kodak) with } 120 \\
\mathrm{kV} \text { and } 36.12 \mathrm{mAs} \text {. } \\
\text { The field of view } \\
\text { (FOV) was } 6 \mathrm{~cm} \\
\text { and the voxel size } \\
\text { was } 0.260 .260 .2 \\
\mathrm{~mm}\end{array}$ & Not available & (-) & 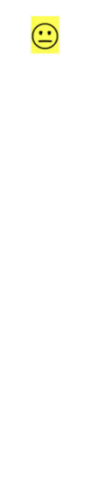 & $\odot$ & $\mathrm{N} / \mathrm{A}$ & (-) & (-) & $\mathrm{N} / \mathrm{A}$ \\
\hline $\begin{array}{l}\text { Fiejo et al. } 2012 \\
\text { [76] }\end{array}$ & $\begin{array}{l}6 \text { patients with } 12 \\
\text { measurement sites }\end{array}$ & $\begin{array}{l}\text { CBCT i-CAT with } \\
\text { voxel size of } 0.2 \\
\mathrm{~mm} \text { and } 40 \\
\text { seconds acquisition } \\
\text { time }\end{array}$ & $\begin{array}{l}\text { Intrasurgical } \\
\text { measurements }\end{array}$ & (-) & (:) & $\oplus$ & :- & (:) & (-) & (:) \\
\hline $\begin{array}{l}\text { Walter et al. } \\
2012 \text { [69] }\end{array}$ & $\begin{array}{l}12 \text { patients, } 3 \\
\text { women and } 9 \\
\text { men, with an } \\
\text { average age of } \\
57.5 \text { years (range: } \\
41-80 \text { years) and a } \\
\text { diagnosis of } \\
\text { generalized } \\
\text { chronic } \\
\text { periodontit-is }\end{array}$ & $\begin{array}{l}\text { CBCT 3D } \\
\text { Accuotom with } \\
\text { volumes of } 494 \\
\mathrm{~cm} \text { to } 696 \mathrm{~cm}\end{array}$ & $\begin{array}{l}\text { Intrasurgical } \\
\text { measurements }\end{array}$ & (-) & (:) & (-) & (:) & (-) & (:) & (-) \\
\hline $\begin{array}{l}\text { Raichur et al. } \\
2012 \text { [81] }\end{array}$ & $\begin{array}{l}7 \text { patient ( } 3 \text { males } \\
\text { and } 4 \text { females) } \\
\text { having moderate } \\
\text { to severe } \\
\text { periodontitis }\end{array}$ & $\begin{array}{l}\text { Digital volume } \\
\text { tomography Kodak } \\
9000 \text { C3D with } \\
\text { (exposure } \\
\text { parameters were } \\
\text { set at 70-74 kV, } 10 \\
\text { mA and } 10.8 \\
\text { seconds\} \& } \\
\text { Radiovisiography } \\
\text { (with a size \#2 } \\
\text { charged couple } \\
\text { device (CCD) } \\
\text { intraoral digital } \\
\text { sensor\$ and a } \\
\text { standard X-ray } \\
\text { unit* operating at } \\
60-63 \text { kV, } 8 \text { mA and }\end{array}$ & $\begin{array}{l}\text { Direct } \\
\text { measurements } \\
\text { with UNC } 15 \\
\text { probe }\end{array}$ & (-) & (:) & ;) & (-) & (:) & (-) & $\odot$ \\
\hline
\end{tabular}


Table 3 Quality assessment of diagnostic accuracy studies using QUADAS2 tool (Continued)

\begin{tabular}{|c|c|c|c|c|c|c|c|c|c|c|}
\hline \multirow[t]{2}{*}{ Study } & \multirow[t]{2}{*}{ Sample } & \multirow[t]{2}{*}{ Index test } & \multirow{2}{*}{$\begin{array}{l}\text { Reference } \\
\text { standard } \\
\text { (comparator) }\end{array}$} & \multicolumn{4}{|c|}{ Risk of bias } & \multicolumn{3}{|c|}{ Applicability concern } \\
\hline & & & & $\begin{array}{l}\text { Patient } \\
\text { Selection }\end{array}$ & $\begin{array}{l}\text { Index } \\
\text { test }\end{array}$ & $\begin{array}{l}\text { Reference } \\
\text { test }\end{array}$ & $\begin{array}{l}\text { Flow and } \\
\text { timing }\end{array}$ & $\begin{array}{l}\text { Patient } \\
\text { selection }\end{array}$ & $\begin{array}{l}\text { Index } \\
\text { test }\end{array}$ & $\begin{array}{l}\text { Reference } \\
\text { standard }\end{array}$ \\
\hline & & $0.25-0.32 \mathrm{sec})$ & & & & & & & & \\
\hline $\begin{array}{l}\text { Marinescu et al. } \\
2013 \text { [84] }\end{array}$ & $\begin{array}{l}19 \text { patients with } \\
\text { (presenting a total } \\
\text { of } 25 \text { lower molars } \\
\text { with different } \\
\text { degrees of } \\
\text { furcation defects ) }\end{array}$ & $\mathrm{CBCT}$ & $\begin{array}{l}\text { Clinical } \\
\text { measurements }\end{array}$ & (:) & (-) & :) & (-) & (-) & (-) & :) \\
\hline $\begin{array}{l}\text { Qiao et al. } 2013 \\
\text { [86] }\end{array}$ & $\begin{array}{l}15 \text { patients ( } 9 \\
\text { women and } 6 \\
\text { men) with an } \\
\text { average age of } \\
43.5 \text { years and a } \\
\text { diagnosis of } \\
\text { generalized } \\
\text { chronic } \\
\text { periodontitis }\end{array}$ & $\begin{array}{l}\text { CBCT Accuitomo } \\
\text { with settings in the } \\
\text { range } 74-90 \mathrm{kV} \\
\text { and } 5-8 \mathrm{~mA} \text { and } \\
\text { voxel size of } 0.125 \\
\times 0.125 \times 0.125 \\
\mathrm{~mm}\end{array}$ & $\begin{array}{l}\text { Intrasurgi-cal } \\
\text { measurem-ents }\end{array}$ & (-) & (-) & 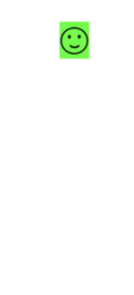 & (-) & (-) & (-) & (-) \\
\hline $\begin{array}{l}\text { Haghgoo et al. } \\
2014 \text { [87] }\end{array}$ & $\begin{array}{l}50 \text { interproximal } \\
\text { sites in patients } \\
\text { having } \\
\text { periodontitis are } \\
\text { assessed }\end{array}$ & $\begin{array}{l}\text { CBCT (Newtom 3G, } \\
\text { Verona. Italy) and } \\
\text { direct digital } \\
\text { intraoral } \\
\text { radiography } \\
\text { (Sopro-La Ciotat- } \\
\text { France) was taken. }\end{array}$ & $\begin{array}{l}\text { Intrasurgi-cal } \\
\text { findings }\end{array}$ & (-) & (-) & (-) & (-) & (-) & (-) & (-) \\
\hline $\begin{array}{l}\text { Banodkar et al. } \\
2015 \text { [88] }\end{array}$ & $\begin{array}{l}15 \text { patients with } \\
\text { hundred } \\
\text { periodontal bone } \\
\text { defects }\end{array}$ & $\begin{array}{l}\text { CBCT promax } \\
\text { (Planmec) with } 90 \\
\mathrm{kv} \text {, voxel size- } 400 \\
\mu \mathrm{m} \text {, current- } 10 \mathrm{~mA} \text {, } \\
\text { and exposure time- } \\
13 \mathrm{~s}\end{array}$ & $\begin{array}{l}\text { Intrasurgical } \\
\text { measurements }\end{array}$ & (-) & (-) & :-) & (-) & $(-)$ & (:) & (-) \\
\hline $\begin{array}{l}\text { Cimbaljevic et al } \\
2015 \text { [89] }\end{array}$ & $\begin{array}{l}15 \text { patients ( } 4 \text { men } \\
\text { and } 11 \text { women } \\
\text { aged } 35 \text { to } 60 \\
\text { years; mean age } \\
44.5 \pm 8.4 \text { years) } \\
\text { with } 174 \text { furcation } \\
\text { sites }\end{array}$ & $\begin{array}{l}\text { CBCT unit } \\
\text { (SCANORA 3Dx, } \\
\text { Soredex) with } 80 \times \\
100 \mathrm{~mm} \text { field of } \\
\text { view, } 0.25 \mathrm{~mm} \\
\text { voxel size, } 90 \mathrm{kV} \\
\text { tube voltage, } 10 \\
\mathrm{~mA} \text { tube current, } \\
\text { and } 2.4 \text { seconds } \\
\text { active scanning } \\
\text { time | Probing } \\
\text { using a Nabers } \\
\text { probe (PQ2N, Hu- } \\
\text { Friedy) }\end{array}$ & Not available & (:) & (:) & N/A & (-) & (:) & (-) & N/A \\
\hline $\begin{array}{l}\text { Darby et al. } \\
2015 \text { [90] }\end{array}$ & $\begin{array}{l}\text { Clinical records ( } \\
\text { Retrospective ) } \\
\text { from the } \\
\text { Periodontics clinic } \\
\text { at the Royal } \\
\text { Dental Hospital of } \\
\text { Melbourne } \\
\text { (RDHM) }\end{array}$ & $\begin{array}{l}\text { CBCT i-CAT with } \\
\text { slice thickness } 1 \\
\mathrm{~mm} \text {, voxel size } 0.2 \\
\mathrm{~mm} .120 \mathrm{kV}, 20.7 \\
\mathrm{mAs}^{-1}, 14.7 \mathrm{~s} \\
\text { acquisition time) }\end{array}$ & Not available & (;) & (-) & N/A & : & (-) & (-) & N/A \\
\hline $\begin{array}{l}\text { Li F et al. } 2015 \\
\text { [91] }\end{array}$ & $\begin{array}{l}44 \text { patients ( } 22 \\
\text { men and } 22 \\
\text { women) with } 44 \\
\text { intrabony defects }\end{array}$ & $\begin{array}{l}\text { CBCT New Tom, } \\
\text { Verona, Italy }(12- \\
17 \mathrm{~mA} \text { and } 110 \mathrm{Kv}) \text {, } \\
\text { and } \\
\text { digitalperiapical } \\
\text { radiography }(70 \mathrm{kVp} \\
\text { and } 12-25 \mathrm{~mA})\end{array}$ & $\begin{array}{l}\text { Intrasurgi-cal } \\
\text { measurements } \\
\text { made with a } \\
\text { probe (HU- } \\
\text { Friedy) }\end{array}$ & (-) & (-) & $\odot$ & (-) & (-) & (-) & (-) \\
\hline
\end{tabular}


Table 3 Quality assessment of diagnostic accuracy studies using QUADAS2 tool (Continued)

\begin{tabular}{|c|c|c|c|c|c|c|c|c|c|c|}
\hline \multirow[t]{2}{*}{ Study } & \multirow[t]{2}{*}{ Sample } & \multirow[t]{2}{*}{ Index test } & \multirow{2}{*}{$\begin{array}{l}\text { Reference } \\
\text { standard } \\
\text { (comparator) }\end{array}$} & \multicolumn{4}{|c|}{ Risk of bias } & \multicolumn{3}{|c|}{ Applicability concern } \\
\hline & & & & $\begin{array}{l}\text { Patient } \\
\text { Selection }\end{array}$ & $\begin{array}{l}\text { Index } \\
\text { test }\end{array}$ & $\begin{array}{l}\text { Reference } \\
\text { test }\end{array}$ & $\begin{array}{l}\text { Flow and } \\
\text { timing }\end{array}$ & $\begin{array}{l}\text { Patient } \\
\text { selection }\end{array}$ & $\begin{array}{l}\text { Index } \\
\text { test }\end{array}$ & $\begin{array}{l}\text { Reference } \\
\text { standard }\end{array}$ \\
\hline $\begin{array}{l}\text { Guo et al. } 2016 \\
\text { [92] }\end{array}$ & $\begin{array}{l}6 \text { patients ( } 2 \text { males } \\
\text { and } 4 \text { females) }\end{array}$ & $\begin{array}{l}\text { CBCT 3D } \\
\text { Accuitomo with a } \\
\text { field of view of } 43 \\
4 \mathrm{~cm} \text {, tube voltage } \\
\text { of } 75-85 \mathrm{kVp} \text { and } \\
\text { tube current of } 5 \\
\mathrm{~mA} \text {. The voxel size } \\
\text { used was } 0.1253 \\
0.12530 .125 \mathrm{~mm} \text {. }\end{array}$ & $\begin{array}{l}\text { Intrasurgical } \\
\text { measurem-ents }\end{array}$ & (;) & (:) & (-) & (-) & () & (-) & (-) \\
\hline $\begin{array}{l}\text { Zhu et al. } 2016 \\
\text { [96] }\end{array}$ & $\begin{array}{l}11 \text { patients (Thirty- } \\
\text { nine sites with } \\
\text { degree } I I \mathrm{Fl} \text {, } \\
\text { classified by } \\
\text { probing of } 21 \\
\text { maxillary molars, } \\
\text { were investigated\} }\end{array}$ & $\begin{array}{l}\text { CBCT 3D } \\
\text { Accuitomo with } \\
\text { volumes of } 4 \times 4 \\
\text { to } 6 \times 6 \mathrm{~cm} \text {, with a } \\
\text { setting in the } \\
\text { range of } 80 \mathrm{kV}, 5.0 \\
\text { to } 6.3 \mathrm{~mA} \text { and a } \\
\text { voxel size of } 0.125 \\
\times 0.125 \times 0.125 \\
\mathrm{~mm}\end{array}$ & Not available & (-) & (-) & N/A & N/A & (-) & (-) & N/A \\
\hline $\begin{array}{l}\text { Suphanantachat } \\
\text { et al.2017 [94] }\end{array}$ & 25 patients & $\begin{array}{l}\text { Intraoral radiograph } \\
\text { (Kodak) with } 70 \mathrm{kV} \text {, } \\
7 \mathrm{~mA} \text {, exposure } \\
\text { time } 0.2-0.4 \mathrm{~s} \mid \\
\mathrm{CBCT} 3 \mathrm{D} \\
\text { Accuitomo with } \\
\text { volumes of } 1003 \\
100 \mathrm{~mm}, 80 \mathrm{kV}, 5 \\
\mathrm{~mA} \text {, exposure time } \\
\text { of } 17.5 \mathrm{~s} \text { and a } \\
\text { voxel size of } 0.25 \\
\mathrm{~mm}\end{array}$ & Not available & (:) & (:) & N/A & (-) & (-) & (-) & N/A \\
\hline $\begin{array}{l}\text { Padmanabhan } \\
\text { et al. } 2017 \text { [95] }\end{array}$ & $\begin{array}{l}14 \text { patients ( } 20-60 \\
\text { years) with } 25 \\
\text { mandibular molar } \\
\text { furcation sites }\end{array}$ & $\begin{array}{l}\text { Intraoral periapical } \\
\text { radiography | CBCT } \\
\text { with } 84 \mathrm{kv}, 5 \mathrm{~mA} \text {, } \\
20 \mathrm{~s} \text {, Voxel size of } \\
180 \mu \mathrm{m}\end{array}$ & $\begin{array}{l}\text { Intrasurgi-cal } \\
\text { measurem-ents }\end{array}$ & (;) & 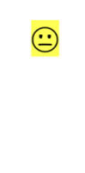 & $\odot$ & (:) & () & (-) & (-) \\
\hline $\begin{array}{l}\text { Zhang et al. } \\
2018 \text { [96] }\end{array}$ & $\begin{array}{l}83 \text { patients with } \\
\text { chronic } \\
\text { periodontitis }\end{array}$ & $\begin{array}{l}\text { CBCT with a field } \\
\text { of view (FOV) of } \\
150 \times 90 \mathrm{~mm} 2 \text {. The } \\
\text { scans were } \\
\text { acquired at } 90 \mathrm{kVp} \text {, } \\
10 \mathrm{~mA}, 16 \mathrm{~s} \text { and a } \\
0.2 \mathrm{~mm} 3 \text { voxel size } \\
\text { with a Kodak } 9500 \\
\text { unit (Carestream } \\
\text { Health, Inc., } \\
\text { Rochester, NY, } \\
\text { USA), Intraoral } \\
\text { using the unit } \\
\text { (Instrumentarium } \\
\text { Dental, Charlotte, } \\
\text { NC, USA) operating } \\
\text { at } 70 \text { kVp, } 7 \text { mA, } \\
\text { and an exposure } \\
\text { time corresponding } \\
\text { to the exposed } \\
\text { area, Clinical } \\
\text { measurements }\end{array}$ & Not available & (-) & (-) & N/A & (-) & (-) & N/A & (:) \\
\hline
\end{tabular}


Table 4 CASP checklist for critical appraisal of randomized clinical trials studies

\begin{tabular}{|c|c|c|c|c|c|c|c|}
\hline Criteria & $\begin{array}{l}\text { Dutra et al. } \\
2017 \text { [82] }\end{array}$ & $\begin{array}{l}\text { Gupta SJ et al. } \\
2014 \text { [72] }\end{array}$ & $\begin{array}{l}\text { Grimrad } \\
\text { et al.2009 [68] }\end{array}$ & $\begin{array}{l}\text { Khosropana } \\
\text { et al.2015 [91] }\end{array}$ & $\begin{array}{l}\text { Nemoto et al. } \\
2018 \text { [85] }\end{array}$ & $\begin{array}{l}\text { Bhavsar et al. } \\
2016 \text { [77] }\end{array}$ & $\begin{array}{l}\text { Pajnigara et al. } \\
2017 \text { [79] }\end{array}$ \\
\hline $\begin{array}{l}\text { Did the trial address a clearly } \\
\text { focused issue? }\end{array}$ & Yes & Yes & Yes & Yes & Yes & Yes & Yes \\
\hline $\begin{array}{l}\text { Was the assignment of patients } \\
\text { to treatments randomized? }\end{array}$ & Yes & Yes & Yes & Yes & Yes & Yes & Yes \\
\hline $\begin{array}{l}\text { Were all of the patients who } \\
\text { entered the trial properly } \\
\text { accounted for at its conclusion? }\end{array}$ & Yes & Yes & Yes & Yes & Yes & Yes & Yes \\
\hline $\begin{array}{l}\text { Were patients, health workers } \\
\text { and study personnel 'blind' to } \\
\text { treatment? }\end{array}$ & Can't tell ${ }^{\mathrm{a}}$ & Can't tell $^{a}$ & Yes & Yes & Yes & Yes & Yes \\
\hline $\begin{array}{l}\text { Were the groups similar at the } \\
\text { start of the trial }\end{array}$ & Yes & Yes & Yes & Yes & Yes & Yes & Yes \\
\hline $\begin{array}{l}\text { Aside from the experimental } \\
\text { intervention, were the groups } \\
\text { treated equally? }\end{array}$ & Yes & Yes & Yes & Yes & Yes & Yes & Yes \\
\hline $\begin{array}{l}\text { How large was the treatment } \\
\text { effect? }\end{array}$ & Satisfactory & Satisfactory & Satisfactory & Satisfactory & Satisfactory & Satisfactory & Satisfactory \\
\hline $\begin{array}{l}\text { How precise was the estimate } \\
\text { of the treatment effect? }\end{array}$ & Acceptable & Acceptable & Acceptable & Accept-able & Acceptable & Acceptable & Acceptable \\
\hline $\begin{array}{l}\text { Can the results be applied to } \\
\text { the local population or in } \\
\text { your contex }\end{array}$ & Yes & Yes & Yes & Yes & Yes & Yes & Yes \\
\hline $\begin{array}{l}\text { Were all clinically important } \\
\text { outcomes considered? }\end{array}$ & Yes & Yes & Yes & Yes & Yes & Yes & Yes \\
\hline $\begin{array}{l}\text { Are the benefits worth the } \\
\text { harms and costs? }\end{array}$ & Yes & Yes & Yes & Yes & Yes & Yes & Yes \\
\hline
\end{tabular}

${ }^{\mathrm{a} C a n}$ 't tell cannot tell; criteria in this tool

and clinical-post surgery CBCT; the three-dimensional imaging gives dental practitioners the chance to optimize treatment decisions and assess the degree of healing more effectively.

\section{Patient's outcome efficacy}

Our systematic review has revealed eight studies that used CBCT to assess the results of treatment provided for periodontal diseases [68, 72, 75, 77, 79, 82, 85, 98]. However, it seems that this study is in disagreement with a previously published review [6]. They did not identify any study on the level of patient outcome. The reason for this could be the difference between our inclusion and exclusion criteria and theirs. All studies agreed that CBCT is a reliable tool in the assessment of the results of treatment using a bone graft.

\section{Societal efficacy}

The study reported by Walter et al. [69] has shown that the use of CBCT decreases the cost and time for periodontitis screening. However, CBCT should only be advised in cases of advanced therapy. Further studies with a sufficient number of patients were suggested.

\section{Systematic reviews}

Our review has resulted in six studies, which are systematic reviews. Each review is supposed to adhere to the criteria provided by AMSTAR and scores YES whenever applicable. The review published by Haas et al. [78] did not elaborate on whether they included the study registries or consulted content experts in the field in terms of comprehensive literature search strategy. Although a meta-analysis was conducted in such a review, the review authors did not assess the potential impact of risk of bias on the results of the meta-analysis or other evidence synthesis. Moreover, the authors did not carry out an adequate investigation of publication bias (smallstudy bias) or discuss its likely impact on the results of the review. Based on our interpretation, the study has not reported any source of funding or mentioned any conflict of interest.

The study by Walter et al. [79] did not clearly have an explicit statement that the review methods were established prior to the conduct of the review and did not justify any significant deviations from the protocol. In addition, only one database has been searched for relevant studies. According to the AMSTAR2 criteria, the included studies were not described adequately. The study has not reported on the source of funding for the 
Table 5 AMSTAR2 checklist for systematic review appraisal

\begin{tabular}{|c|c|c|c|c|c|c|}
\hline \multirow[t]{2}{*}{ Criteria } & \multicolumn{6}{|c|}{ Systematic Reviews } \\
\hline & $\begin{array}{l}\text { Haas et al. } \\
2018 \text { [78] }\end{array}$ & $\begin{array}{l}\text { Anter et al. } \\
2016 \text { [73] }\end{array}$ & $\begin{array}{l}\text { Walter et al. } \\
2016[70]\end{array}$ & $\begin{array}{l}\text { Nikolic-Jakoba } \\
\text { et al. } \\
2016 \text { [6] }\end{array}$ & $\begin{array}{l}\text { Choi et al. } \\
2018 \text { [80] }\end{array}$ & $\begin{array}{l}\text { Woelber et al. } \\
2018[83]\end{array}$ \\
\hline $\begin{array}{l}\text { 1. Did the research questions and inclusion } \\
\text { criteria for the review include the } \\
\text { components of PICO? }\end{array}$ & Yes & No & Yes & Yes & Yes & Yes \\
\hline $\begin{array}{l}\text { 2. Did the report of the review contain an } \\
\text { explicit statement that the review methods } \\
\text { were established prior to the conduct of } \\
\text { the review and did the report justify any } \\
\text { significant deviations from the protocol? }\end{array}$ & Yes & Yes & Partial yes & Yes & Partial yes & Yes \\
\hline $\begin{array}{l}\text { 3. Did the review authors explain their } \\
\text { reasons for selection of the study designs } \\
\text { for inclusion in the review? }\end{array}$ & Yes & Yes & Yes & Yes & $\begin{array}{l}\text { No } \\
\text { "Some failed to } \\
\text { continue" }\end{array}$ & Yes \\
\hline $\begin{array}{l}\text { 4. Did the review authors use a } \\
\text { comprehensive literature search strategy? }\end{array}$ & Partial yes & Partial yes & No & Yes & Yes & Yes \\
\hline $\begin{array}{l}\text { 5. Did the review authors perform study } \\
\text { selection in duplicate? }\end{array}$ & Yes & Yes & No & Yes & Yes & Yes \\
\hline $\begin{array}{l}\text { 6. Did the review authors perform data } \\
\text { extraction in duplicate? }\end{array}$ & Yes & No & Yes & Yes & Yes & Yes \\
\hline $\begin{array}{l}\text { 7. Did the review authors provide a list of } \\
\text { excluded studies and justify the exclusions? }\end{array}$ & Yes & Yes & Yes & Partial yes & Partial yes & Yes \\
\hline $\begin{array}{l}\text { 8. Did the review authors describe the } \\
\text { included studies in adequate detail? }\end{array}$ & Yes & Partial yes & Partial yes & Yes & Yes & Yes \\
\hline $\begin{array}{l}\text { 9. Did the review authors use a satisfactory } \\
\text { technique for assessing the risk of bias (RoB) } \\
\text { in individual studies included in the review? }\end{array}$ & Yes & Partial yes & No & Yes & No & No \\
\hline $\begin{array}{l}\text { 10. Did the review authors report on the } \\
\text { sources of funding for the studies included } \\
\text { in the review? }\end{array}$ & No & No & No & No & No & No \\
\hline $\begin{array}{l}\text { 11. If meta-analysis was performed, did the } \\
\text { review authors use appropriate methods } \\
\text { for a statistical combination of results? }\end{array}$ & Yes & $\begin{array}{l}\text { No meta-analysis } \\
\text { conducted }\end{array}$ & $\begin{array}{l}\text { No meta- } \\
\text { analysis }\end{array}$ & $\begin{array}{l}\text { No meta-analysis } \\
\text { conduct-ed }\end{array}$ & $\begin{array}{l}\text { No meta- } \\
\text { analysis }\end{array}$ & $\begin{array}{l}\text { No meta- } \\
\text { analysis } \\
\text { performed }\end{array}$ \\
\hline $\begin{array}{l}\text { 12. If meta-analysis was performed, did the } \\
\text { review authors assess the potential impact } \\
\text { of RoB on individual studies based on the } \\
\text { results of the meta-analysis or other } \\
\text { evidence synthesis? }\end{array}$ & No & $\begin{array}{l}\text { No meta-analysis } \\
\text { conducted }\end{array}$ & $\begin{array}{l}\text { No meta- } \\
\text { analysis }\end{array}$ & $\begin{array}{l}\text { No meta-analysis } \\
\text { conducted }\end{array}$ & $\begin{array}{l}\text { No meta- } \\
\text { analysis } \\
\text { performed }\end{array}$ & $\begin{array}{l}\text { No meta- } \\
\text { analysis } \\
\text { performed }\end{array}$ \\
\hline $\begin{array}{l}\text { 13. Did the review authors account for RoB } \\
\text { in individual studies when interpreting/ } \\
\text { discussing the results of the review? }\end{array}$ & Yes & Yes & $\begin{array}{l}\mathrm{No}^{\mathrm{a}} \\
\text { "No RoB } \\
\text { assessed" }\end{array}$ & Yes & No & No \\
\hline $\begin{array}{l}\text { 14. Did the review authors provide a } \\
\text { satisfactory explanation for, and discussion } \\
\text { of, any heterogeneity observed in the results } \\
\text { of the review? }\end{array}$ & Yes & Yes & Yes & Yes & Yes & Yes \\
\hline $\begin{array}{l}\text { 15. If they performed quantitative synthesis, } \\
\text { did the review authors carry out an } \\
\text { adequate investigation of publication bias } \\
\text { (small study bias) and discuss its likely } \\
\text { impact on the results of the review? }\end{array}$ & No & $\begin{array}{l}\text { No meta- } \\
\text { analy-sis } \\
\text { cond-ucted }\end{array}$ & $\begin{array}{l}\text { No meta-analysis } \\
\text { conduct-ed }\end{array}$ & $\begin{array}{l}\text { No meta-analysis } \\
\text { conduc-ted }\end{array}$ & $\begin{array}{l}\text { No meta- } \\
\text { analysis } \\
\text { performed }\end{array}$ & $\begin{array}{l}\text { No meta- } \\
\text { analysis } \\
\text { perform-ed }\end{array}$ \\
\hline $\begin{array}{l}\text { 16. Did the review authors report any } \\
\text { potential sources of conflict of interest, } \\
\text { including any funding they received for } \\
\text { conducting }\end{array}$ & No & Yes & No & Yes & No & Yes \\
\hline
\end{tabular}

${ }^{\mathrm{a}}$ No $R O B$ no risk of bias assessed 
individual studies included in the review. To our knowledge, the risk of bias has not been elaborated upon in the relevant sites in the review. Moreover, the review authors did not account for the risk of bias in individual studies when interpreting or discussing the results of the review. In addition, the authors have not reported any source of conflict including any funding they received for conducting the review.

The review by Anter et al. addressed the accuracy of the CBCT as a tool for the measurement of alveolar bone loss in periodontal defects. However, the authors did not report that they followed PICO, which is a framework for review question formulation [36]. In terms of a comprehensive search strategy, we saw that this review did not fulfill the criteria regarding study registries and expert consultation in the field. Furthermore, the authors did not conduct the search in duplicate for the purpose of study selection. The review authors had also not performed data extraction in duplicates. According to our interpretation, the included studies were not described in appropriate detail. Additionally, the source of funding for each relevant individual study was not reported.

The study reported by Choi et al. [80] did not specify whether if there was a deviation from protocol, metaanalysis plan, or causes of heterogeneity if appropriate. In addition, a list of the excluded study in association with a justification for exclusion of each potential study has not been provided. Regardless of whether it is one of the targets of the review, this review has not discussed any potential risk of bias of the included studies. Moreover, the source of funding of each included study was also not reported. It could be included that this review does fulfill the AMSTAR2 [97] checklist to some extent.

The review by Woelber et al. [83] neither mentions any deviation from protocol whenever applicable nor elaborates on if is a plan for meta-analysis, if appropriate. In addition, a plan for investigating the possible causes, if appropriate, regarding heterogeneity was also not reported. The source of funding for each included study was not reported either. To some extent, the review fulfills the checklist of AMSTAR2.

According to our systematic review and AMSTAR2 tool, we found the review conducted by Nikolic-Jakoba et al. [6] best fulfills the tool criteria. However, the study's authors did not justify the reason for exclusion of each potentially relevant study from the review. As other reviews were included in our study, the source of funding of each included publication was not reported.

\section{Conclusion}

We concluded that most of the studies conducted on the rule of $\mathrm{CBCT}$ in periodontal diseases were at diagnostic accuracy level followed by the patient outcome level. Accordingly, it was found that $\mathrm{CBCT}$ is quite beneficial and accurate in the diagnosis of infra-bony defects and furcation involvement. Similarly, it is reliable in the assessment of the outcome of periodontal surgery and regenerative therapy. Furthermore, more studies with a larger cohort on the level of diagnostic thinking, therapeutic, and societal efficacy are needed to set up a clear guideline and evidence for the usefulness of CBCT.

\begin{abstract}
Abbreviations
CBCT: Cone beam computed tomography; CT: Computed tomography; PRISMA: Preferred items for systematic review and meta-analysis; CRD: Center for reviews and dissemination; PICO: Population, or Problem, Intervention or Exposure, Comparison (if appropriate), Outcome you would like to measure or achieve; QUADAS2: Quality assessment tool for diagnostic accuracy studies; CASP: Critical appraisal skills program; AMSTAR: A measurement tool to assess systematic review; 2D: Two-dimensional; 3D: Three-dimensional
\end{abstract}

\section{Acknowledgments}

No other contributors to acknowledge.

\section{Authors' contributions}

HA: Literature search, analyzing individual studies, manuscript preparation and review; AAD: Literature search, analyzing individual studies, manuscript review; AA: Analyzing individual studies, manuscript review; ZA: Literature search, manuscript review. The author(s) read and approved the final manuscript.

\section{Funding}

No funding was provided for this systematic review.

\section{Availability of data and materials}

The datasets used and/or analyzed during the current study are available from the corresponding author on reasonable request.

\section{Ethics approval and consent to participate}

The research committee at the faculty of dentistry in King Khalid University has approved the study proposal. Since it is a review, the consent of participants is not applicable.

\section{Consent for publication}

Not applicable.

\section{Competing interests}

The authors declare they have no competing interests.

\section{Author details}

'Department of Oral Biology and Diagnostic Science, King Khalid University, College of Dentistry, Abha, Saudi Arabia. ${ }^{2}$ Department of Periodontology, King Khalid University, College of Dentistry, Abha, Saudi Arabia. ${ }^{3}$ College of Dentistry, King Khalid University, Abha, Saudi Arabia.

Received: 21 November 2019 Accepted: 5 April 2020

Published online: 08 July 2020

\section{References}

1. Flemmig TF. Periodontitis. Ann Periodontol. 1999;4(1):32-8.

2. Highfield J. Diagnosis and classification of periodontal disease. Aust Dent J. 2009:54(Suppl 1):S11-26.

3. Pihlstrom BL, Michalowicz BS, Johnson NW. Periodontal diseases. Lancet (London, England). 2005:366(9499):1809-20.

4. Loe H, Anerud A, Boysen H, Morrison E. Natural history of periodontal disease in man. Rapid, moderate and no loss of attachment in Sri Lankan laborers 14 to 46 years of age. J Clin Periodontol. 1986;13(5):431-45.

5. Burt B. Position paper: epidemiology of periodontal diseases. J Periodontol. 2005;76(8):1406-19.

6. Nikolic-Jakoba N, Spin-Neto R, Wenzel A. Cone beam computed tomography for detection of Intrabony and furcation defects: a Systematic 
review based on a hierarchical model for diagnostic efficacy. J Periodontol. 2016;87(6):630-44.

7. Jordan RCK. Diagnosis of periodontal manifestations of systemic diseases. Periodontol 2000. 2004:34:217-29.

8. Listgarten MA. Periodontal probing: what does it mean? J Clin Periodontol. 1980;7(3):165-76.

9. Tugnait A, Clerehugh V, Hirschmann PN. The usefulness of radiographs in diagnosis and management of periodontal diseases: a review. J Dent. 2000; 28(4):219-26.

10. Tyndall DA, Rathore S. Cone beam CT diagnostic applications: caries, periodontal bone assessment, and endodontic applications. Dent Clin N Am. 2008;52(4):825-41 vii.

11. Braun X, Ritter L, Jervoe-Storm P-M, Frentzen M. Diagnostic accuracy of CBCT for periodontal lesions. Clin Oral Investig. 2014;18(4):1229-36.

12. Summers A. Gingivitis: diagnosis and treatment. Emerg Nurse. 2009;17(1): 18-20 quiz 35.

13. Bailey DL, Barrow S-Y, Cvetkovic B, Musolino R, Wise SL, Yung C, et al. Periodontal diagnosis in private dental practice: a case-based survey. Aust Dent J. 2016;61(2):244-51.

14. Garcia-Pola M-J, Rodriguez-Lopez S, Fernanz-Vigil A, Bagan L, Garcia-Martin J-M. Oral hygiene instructions and professional control as part of the treatment of desquamative gingivitis. Systematic review. Med Oral Patol Oral Cir Bucal. 2019;24(2):e136-44.

15. Socransky SS, Haffajee AD. The bacterial etiology of destructive periodontal disease: current concepts. J Periodontol. 1992;63(4 Suppl):322-31.

16. Armitage GC. The complete periodontal examination. Periodontol 2000. 2004;34:22-33.

17. Taba MJ, Kinney J, Kim AS, Giannobile WV. Diagnostic biomarkers for oral and periodontal diseases. Dent Clin N Am. 2005;49(3):551-71 vi.

18. Wolf DL, Lamster IB. Contemporary concepts in the diagnosis of periodontal disease. Dent Clin N Am. 2011;55(1):47-61.

19. Albandar JM, Brunelle JA, Kingman A. Destructive periodontal disease in adults 30 years of age and older in the United States, 1988-1994. J Periodontol. 1999;70(1):13-29.

20. Facial radiology (Evidence-based guidelines) ISSN 1681-6803 MJ-XA-12-001EN-C Energy Protection Radiation No 172 Cone beam CT for dental and maxillofacial radiology (Evidence-based guidelines) [Internet]. 2012. Available from: http://www.sedentexct.eu/files/radiation_protection_172.pdf.

21. Offenbacher S. Periodontal diseases: pathogenesis. Ann Periodontol. 1996; 1(1):821-78.

22. Ozmeric N, Kostioutchenko I, Hagler G, Frentzen M, Jervoe-Storm P-M. Cone beam computed tomography in assessment of periodontal ligament space: in vitro study on artificial tooth model. Clin Oral Investig. 2008;12(3):233-9.

23. Hashimoto K, Kawashima S, Araki M, Iwai K, Sawada K, Akiyama Y. Comparison of image performance between cone beam computed tomography for dental use and four-row multidetector helical CT. J Oral Sci. 2006;48(1):27-34.

24. Loubele M, Maes F, Schutyser F, Marchal G, Jacobs R, Suetens P. Assessment of bone segmentation quality of cone beam $\mathrm{CT}$ versus multislice spiral $\mathrm{CT}$ : a pilot study. Oral Surg Oral Med Oral Pathol Oral Radiol Endod. 2006;102(2):225-34.

25. Shukla S, Chug A, Afrashtehfar Kl. Role of cone beam computed tomography in diagnosis and treatment planning in dentistry: an update. J Int Soc Prev Community Dent. 2017;7(Suppl 3):S125-36.

26. Orentlicher G, Goldsmith D, Abboud M. Computer-guided planning and placement of dental implants. Atlas Oral Maxillofac Surg Clin North Am. 2012;20(1):53-79.

27. Mohan R, Singh A, Gundappa M. Three-dimensional imaging in periodontal diagnosis - utilization of cone beam computed tomography. J Indian Soc Periodontol. 2011;15(1):11-7.

28. Takeshita WM, Vessoni Iwaki LC, Da Silva MC, Tonin RH. Evaluation of diagnostic accuracy of conventional and digital periapical radiography, panoramic radiography, and cone beam computed tomography in the assessment of alveolar bone loss. Contemp Clin Dent. 2014;5(3):318-23.

29. Jeffcoat MK. Radiographic methods for the detection of progressive alveolar bone loss. J Periodontol. 1992;63(4 Suppl):367-72.

30. Swennen GRJ, Mommaerts MY, Abeloos J, De Clerca C, Lamoral P, Neyt N, et al. A cone beam $C T$ based technique to augment the $3 \mathrm{D}$ virtual skull model with a detailed dental surface. Int J Oral Maxillofac Surg. 2009;38(1):48-57.

31. Worthington P, Rubenstein J, Hatcher DC. The role of cone beam computed tomography in the planning and placement of implants. J Am Dent Assoc. 2010;141(Suppl):19S-24S.
32. Alqerban A, Hedesiu M, Baciut M, Nackaerts O, Jacobs R, Fieuws $\mathrm{S}$, et al. Presurgical treatment planning of maxillary canine impactions using panoramic vs cone beam CT imaging. Dentomaxillofac Radiol. 2013;42(9):20130157.

33. Fryback DG, Thornbury JR. The efficacy of diagnostic imaging. Med Decis Mak. 1991;11(2):88-94.

34. Moher D, Liberati A, Tetzlaff J, Altman DG. Preferred reporting items for systematic reviews and meta-analyses: the PRISMA statement. PLoS Med. 2009;6(7):e1000097.

35. Tacconelli E. Systematic reviews: CRD's guidance for undertaking reviews in health care. Lancet Infect Dis. 2010;10(4):226. https://doi.org/10.1016/S14733099(10)70065-7.

36. Speckman RA, Friedly JL. Asking structured, answerable clinical questions using the population, intervention/comparator, outcome (PICO) framework: PM R; 2019.

37. Whiting PF, Rutjes AWS, Westwood ME, Mallett S, Deeks JJ, Reitsma JB, et al. QUADAS-2: a revised tool for the quality assessment of diagnostic accuracy studies. Ann Intern Med. 2011;155(8):529-36.

38. Bagis N, Kolsuz ME, Kursun S, Orhan K. Comparison of intraoral radiography and cone beam computed tomography for the detection of periodontal defects: an in vitro study. BMC Oral Health. 2015;15:64.

39. Almeida VC, Pinheiro LR, Salineiro FCS, Mendes FM, Neto JBC, Cavalcanti MGP, et al. Performance of cone beam computed tomography and conventional intraoral radiographs in detecting interproximal alveolar bone lesions: a study in pig mandibles. BMC Oral Health. 2017;17(1):100.

40. Fleiner J, Hannig C, Schulze D, Stricker A, Jacobs R. Digital method for quantification of circumferential periodontal bone level using cone beam CT. Clin Oral Investig. 2013;17(2):389-96.

41. Kamburoglu K, Kolsuz E, Murat S, Eren H, Yuksel S, Paksoy CS. Assessment of buccal marginal alveolar peri-implant and periodontal defects using a cone beam CT system with and without the application of metal artefact reduction mode. Dentomaxillofac Radiol. 2013;42(8):20130176.

42. Misch KA, Yi ES, Sarment DP. Accuracy of cone beam computed tomography for periodontal defect measurements. J Periodontol. 2006;77(7):1261-6.

43. Mol A, Balasundaram A. In vitro cone beam computed tomography imaging of periodontal bone. Dentomaxillofac Radiol. 2008;37(6):319-24.

44. Noujeim M, Prihoda T, Langlais R, Nummikoski P. Evaluation of highresolution cone beam computed tomography in the detection of simulated interradicular bone lesions. Dentomaxillofac Radiol. 2009;38(3):156-62.

45. Salineiro FCS, Gialain IO, Kobayashi-Velasco S, Pannuti CM, Cavalcanti MGP. Detection of furcation involvement using periapical radiography and 2 cone beam computed tomography imaging protocols with and without a metallic post: an animal study. Imaging Sci Dent. 2017;47(1):17-24.

46. Vandenberghe B, Jacobs R, Yang J. Detection of periodontal bone loss using digital intraoral and cone beam computed tomography images: an in vitro assessment of bony and/or infrabony defects. Dentomaxillofac Radiol. 2008;37(5):252-60.

47. Vandenberghe $B$, Jacobs $R$, Yang J. Diagnostic validity (or acuity) of $2 D C C D$ versus 3D CBCT-images for assessing periodontal breakdown. Oral Surg Oral Med Oral Pathol Oral Radiol Endod. 2007;104(3):395-401.

48. Kolsuz ME, Bagis N, Orhan K, Avsever H, Demiralp KO. Comparison of the influence of FOV sizes and different voxel resolutions for the assessment of periodontal defects. Dentomaxillofac Radiol. 2015;44(7):20150070.

49. Kamburoglu K, Yeta EN, Yilmaz F. An ex vivo comparison of diagnostic accuracy of cone beam computed tomography and periapical radiography in the detection of furcal perforations. J Endod. 2015;41(5):696-702.

50. Elashiry M, Meghil MM, Kalathingal S, Buchanan A, Elrefai R, Looney S, et al. Application of radiopaque micro-particle fillers for 3-D imaging of periodontal pocket analogues using cone beam CT. Dent Mater. 2018;34(4):619-28.

51. Lim H-C, Jeon S-K, Cha J-K, Lee J-S, Choi S-H, Jung U-W. Prevalence of cervical enamel projection and its impact on furcation involvement in mandibular molars: a cone beam computed tomography study in Koreans. Anat Rec (Hoboken). 2016;299(3):379-84.

52. Ozcan G, Sekerci AE. Classification of alveolar bone destruction patterns on maxillary molars by using cone beam computed tomography. Niger J Clin Pract. 2017;20(8):1010-9.

53. Yang $\mathrm{Y}$, Yang $\mathrm{H}$, Pan $\mathrm{H}, \mathrm{Xu}$ J, Hu T. Evaluation and new classification of alveolar bone dehiscences using cone beam computed tomography in vivo. Int J Morphol. 2015;33:361-8.

54. Amorfini L, Migliorati M, Signori A, Silvestrini-Biavati A, Benedicenti S. Block allograft technique versus standard guided bone regeneration: a randomized clinical trial. Clin Implant Dent Relat Res. 2014;16(5):655-67. 
55. Alshaer S, Alhaffar I, Khattab R. Evaluating change in radiographic bone density via cone beam computed tomography before and after surgery to patients with chronic periodontitis. Int J Pharmaceutical Sci Rev Res. 2013; 23:143-7.

56. Al-Zahrani MS, Elfirt EY, Al-Ahmari MM, Yamany IA, Alabdulkarim MA, Zawawi $\mathrm{KH}$. Comparison of cone beam computed tomography-derived alveolar bone density between subjects with and without aggressive periodontitis. J Clin Diagn Res. 2017;11(1):ZC118-21.

57. Ferreira PP, Torres M, Campos PSF, Vogel CJ, de Araujo TM, Rebello IMCR. Evaluation of buccal bone coverage in the anterior region by cone beam computed tomography. Am J Orthod Dentofac Orthop. 2013;144(5):698704.

58. Evangelista K, de Faria VK, Bumann A, Hirsch E, Nitka M, Silva MAG. Dehiscence and fenestration in patients with Class I and Class II Division 1 malocclusion assessed with cone beam computed tomography. Am J Orthod Dentofacial Orthop. 2010;138(2):133.e1-7 discussion 133-5.

59. Sun L, Zhang L, Shen G, Wang B, Fang B. Accuracy of cone beam computed tomography in detecting alveolar bone dehiscences and fenestrations. Am J Orthod Dentofac Orthop. 2015:147(3):313-23.

60. Leung CC, Palomo L, Griffith R, Hans MG. Accuracy and reliability of cone beam computed tomography for measuring alveolar bone height and detecting bony dehiscences and fenestrations. Am J Orthod Dentofac Orthop. 2010;137(4 Suppl):S109-19.

61. Goodarzi Pour D, Romoozi E, Soleimani SY. Accuracy of cone beam computed tomography for detection of bone loss. J Dent (Tehran). 2015; 12(7):513-23.

62. Nagao J, Mori K, Kitasaka T, Suenaga Y, Yamada S, Naitoh M. Quantification and visualization of alveolar bone resorption from 3D dental CT images. Int J Computer Assisted Radiol Surg. 2007;2:43-53.

63. Naitoh M, Yamada S, Noguchi T, Ariji E, Nagao J, Mori K, et al. Threedimensional display with quantitative analysis in alveolar bone resorption using cone beam computerized tomography for dental use: a preliminary study. Int J Periodontics Restorative Dent. 2006;26(6):607-12.

64. Deng Y, Wang C, Li T, Li A, Gou J. An application of cone beam CT in the diagnosis of bone defects for chronic periodontitis. Zhonghua Kou Qiang Yi Xue Za Zhi. 2015;50(1):7-12.

65. Nagao J, Kitasaka T, Mori K, Suenaga Y, Shohzoh Yamada MN. Threedimensional analysis of alveolar bone resorption by image processing of 3D dental CT images. J Med Imaging. 2006;6144.

66. Walter C, Kaner D, Berndt DC, Weiger R, Zitzmann NU. Three-dimensional imaging as a pre-operative tool in decision making for furcation surgery. J Clin Periodontol. 2009;36(3):250-7.

67. Pajnigara N, Kolte A, Kolte R, Pajnigara N, Lathiya V. Diagnostic accuracy of cone beam computed tomography in identification and postoperative evaluation of furcation defects. J Indian Soc Periodontol. 2016;20(4):386-90

68. Grimard BA, Hoidal MJ, Mills MP, Mellonig JT, Nummikoski PV, Mealey BL. Comparison of clinical, periapical radiograph, and cone beam volume tomography measurement techniques for assessing bone level changes following regenerative periodontal therapy. J Periodontol. 2009;80(1):48-55.

69. Walter $C$, Weiger R, Dietrich T, Lang NP, Zitzmann NU. Does threedimensional imaging offer a financial benefit for treating maxillary molars with furcation involvement? A pilot clinical case series. Clin Oral Implants Res. 2012;23(3):351-8.

70. Walter C, Schmidt JC, Dula K, Sculean A. Cone beam computed tomography $(\mathrm{CBCT})$ for diagnosis and treatment planning in periodontology: a systematic review. Quintessence Int. 2016;47(1):25-37.

71. Walter C, Weiger R, Zitzmann NU. Accuracy of three-dimensional imaging in assessing maxillary molar furcation involvement. J Clin Periodontol. 2010; 37(5):436-41.

72. Gupta SJ, Jhingran R, Gupta V, Bains VK, Madan R, Rizvi I. Efficacy of plateletrich fibrin vs. enamel matrix derivative in the treatment of periodontal intrabony defects: a clinical and cone beam computed tomography study. J Int Acad Periodontol. 2014;16(3):86-96.

73. Anter E, Zayet MK, El-Dessouky SH. Accuracy and precision of cone beam computed tomography in periodontal defects measurement (systematic review). J Indian Soc Periodontol. 2016;20(3):235-43.

74. de Faria VK, Evangelista KM, Rodrigues CD, Estrela C, de Sousa TO, Silva MAG. Detection of periodontal bone loss using cone beam CT and intraoral radiography. Dentomaxillofac Radiol. 2012;41(1):64-9.

75. Khosropanah H, Shahidi S, Basri A, Houshyar M. Treatment of Intrabony defects by DFDBA alone or in combination with PRP: a Split-mouth randomized clinical and three-dimensional radiographic trial. J Dent (Tehran). 2015;12(10):764-73.

76. Feijo CV, de Lucena JGF, Kurita LM, Pereira SL da S. Evaluation of cone beam computed tomography in the detection of horizontal periodontal bone defects: an in vivo study. Int J Periodontics Restorative Dent. 2012; 32(5):e162-8.

77. Bhavsar NV, Trivedi SR, Dulani K, Brahmbhatt N, Shah S, Chaudhri D. Clinical and radiographic evaluation of effect of risedronate $5 \mathrm{mg}$ as an adjunct to treatment of chronic periodontitis in postmenopausal women (12-month study). Osteoporos Int. 2016;27(8):2611-9.

78. Haas LF, Zimmermann GS, De Luca CG, Flores-Mir C, Correa M. Precision of cone beam $C T$ to assess periodontal bone defects: a systematic review and meta-analysis. Dentomaxillofac Radiol. 2018:47(2):20170084.

79. Pajnigara NG, Kolte AP, Kolte RA, Pajnigara NG. Volumetric assessment of regenerative efficacy of demineralized freeze-dried bone allograft with or without amnion membrane in grade II furcation defects: a cone beam computed tomography study. Int J Periodontics Restorative Dent. 2017; 37(2):255-62.

80. Choi IGG, Cortes ARG, Arita ES, Georgetti MAP. Comparison of conventional imaging techniques and CBCT for periodontal evaluation: a systematic review. Imaging Sci Dent. 2018:48(2):79-86.

81. Raichur PS, Setty SB, Thakur SL, Naikmasur VG. Comparison of radiovisiography and digital volume tomography to direct surgical measurements in the detection of infrabony defects. J Clin Exp Dent. 2012; 4(1):e43-7.

82. Dutra BC, Oliveira AMSD, Oliveira PAD, Manzi FR, Cortelli SC, de Miranda $\mathrm{CLSSO}$, et al. Effect of $1 \%$ sodium alendronate in the non-surgical treatment of periodontal intraosseous defects: a 6-month clinical trial. J Appl Oral Sci. 2017;25(3):310-7.

83. Woelber JP, Fleiner J, Rau J, Ratka-Kruger P, Hannig C. Accuracy and usefulness of CBCT in periodontology: a Systematic review of the literature. Int J Periodontics Restorative Dent. 2018;38(2):289-97.

84. George Marinescu A, Boariu M, Rusu D, Stratul S-I, Ogodescu A. Reliability of CBCT as an assessment tool for mandibular molars furcation defects. Prog Biomed Opt Imaging - Proc SPIE. 2013;8925.

85. Nemoto Y, Kubota T, Nohno K, Nezu A, Morozumi T, Yoshie H. Clinical and $\mathrm{CBCT}$ evaluation of combined periodontal regenerative therapies using enamel matrix derivative and Deproteinized bovine bone mineral with or without collagen membrane. Int J Periodontics Restorative Dent. 2018;38(3): 373-81.

86. Qiao J, Wang S, Duan J, Zhang Y, Qiu Y, Sun C, et al. The accuracy of cone beam computed tomography in assessing maxillary molar furcation involvement. J Clin Periodontol. 2014;41(3):269-74.

87. Moradi Haghgoo J, Shokri A, Khodadoustan A, Khoshhal M, Rabienejad N, Farhadian M. Comparison the accuracy of the cone beam computed tomography with digital direct intraoral radiography, in assessment of periodontal osseous lesions. Avicenna J Dent Res. 2014;6.

88. Banodkar AB, Gaikwad RP, Gunjikar TU, Lobo TA. Evaluation of accuracy of cone beam computed tomography for measurement of periodontal defects: a clinical study. J Indian Soc Periodontol. 2015;19(3):285-9.

89. Cimbaljevic MM, Spin-Neto RR, Miletic VJ, Jankovic SM, Aleksic ZM, NikolicJakoba NS. Clinical and CBCT-based diagnosis of furcation involvement in patients with severe periodontitis. Quintessence Int. 2015:46(10):863-70.

90. Darby I, Sanelli M, Shan S, Silver J, Singh A, Soedjono M, et al. Comparison of clinical and cone beam computed tomography measurements to diagnose furcation involvement. Int J Dent Hyg. 2015;13(4):241-5.

91. Li F, Jia PY, Ouyang XY. Comparison of measurements on cone beam computed tomography for periodontal Intrabony defect with intra-surgical measurements. Chin J Dent Res. 2015:18(3):171-6.

92. Guo Y-J, Ge Z, Ma R, Hou J, Li G. A six-site method for the evaluation of periodontal bone loss in cone beam CT images. Dentomaxillofac Radiol. 2016:45(1):20150265.

93. Zhu J, Ouyang XY. Assessing maxillary molar furcation involvement by cone beam computed tomography. Chin J Dent Res. 2016;19(3):145-51.

94. Suphanantachat S, Tantikul K, Tamsailom S, Kosalagood P, Nisapakultorn $\mathrm{K}$, Tavedhikul K. Comparison of clinical values between cone beam computed tomography and conventional intraoral radiography in periodontal and infrabony defect assessment. Dentomaxillofac Radiol. 2017;46(6):20160461.

95. Padmanabhan S, Dommy A, Guru SR, Joseph A. Comparative evaluation of cone beam computed tomography versus direct surgical measurements in 
the diagnosis of mandibular molar furcation involvement. Contemp Clin Dent. 2017;8(3):439-45.

96. Zhang W, Foss K, Wang B-Y. A retrospective study on molar furcation assessment via clinical detection, intraoral radiography and cone beam computed tomography. BMC Oral Health. 2018;18(1):75.

97. Zeng X, Zhang Y, Kwong JSW, Zhang C, Li S, Sun F, et al. The methodological quality assessment tools for preclinical and clinical studies, systematic review and meta-analysis, and clinical practice guideline: a systematic review. J Evid Based Med. 2015;8(1):2-10.

98. Gupta V, Bains VK, Singh GP, Jhingran R. Clinical and cone beam computed tomography comparison of NovaBone dental putty and PerioGlas in the treatment of mandibular class II furcations. Indian J Dent Res. 2014;25(2): 166-73.

\section{Publisher's Note}

Springer Nature remains neutral with regard to jurisdictional claims in published maps and institutional affiliations.

Ready to submit your research? Choose BMC and benefit from:

- fast, convenient online submission

- thorough peer review by experienced researchers in your field

- rapid publication on acceptance

- support for research data, including large and complex data types

- gold Open Access which fosters wider collaboration and increased citations

- maximum visibility for your research: over $100 \mathrm{M}$ website views per year

At BMC, research is always in progress.

Learn more biomedcentral.com/submissions 